\title{
Wolbachia and cytoplasmic incompatibility in mycophagous Drosophila and their relatives
}

\author{
JOHN H. WERREN* \& JOHN JAENIKE \\ Department of Biology, University of Rochester, Rochester, New York 14627, U.S.A.
}

\begin{abstract}
Bacterial symbionts belonging to the genus Wolbachia are associated with postzygotic reproductive incompatibility in a number of insect species. Using polymerase chain reaction (PCR) amplification of bacterial gene sequences, strains from 10 species belonging to the closely related quinaria, testacea and tripunctata groups of Drosophila were screened for the presence of Wolbachia in their reproductive tissues. Those screened included the mycophagous species $D$. falleni, $D$. recens, $D$. phalerata, D. testacea, D. neotestacea, D. orientacea, D. putrida and D. tripunctata, and the nonmycophagous species $D$. palustris and $D$. quinaria. Two species, $D$. recens and $D$. orientacea, were found to be infected with Wolbachia. Subsequent tests of four additional strains of $D$. recens found all to be infected with the bacteria. It was established that these bacteria cause partial cytoplasmic incompatibility in $D$. recens by antibiotic curing followed by crosses between cured and uncured strains. Curing was confirmed by a PCR assay. Although most species of insects shown to be infected with Wolbachia are cosmopolitan and/or have undergone recent range expansion in association with human activity, $D$. recens and $D$. orientacea are endemic species with specialized ecological habits. Preliminary molecular phylogenetic analysis indicates that, among the species we examined, $D$. quinaria is most closely related to $D$. recens. To determine whether the bacteria are involved in reproductive isolation between these two species, reciprocal crosses were carried out between $D$. quinaria and both infected and uninfected (cured) strains of $D$. recens. Although these species did mate with each other, all interspecific crosses failed to yield hybrid progeny, indicating that the bacteria are not responsible for reproductive incompatibility between these species.
\end{abstract}

Keywords: cytoplasmic incompatibility, mycophagous Drosophila, Wolbachia.

\section{Introduction}

Wolbachia are a group of closely related bacteria that infect the reproductive tissues of various arthropods. These bacteria are transmitted through the egg cytoplasm and alter reproduction in their arthropod hosts in ways that promote the spread of the bacteria through host populations. So far, Wolbachia have been found to be associated with postzygotic reproductive incompatibility (termed cytoplasmic incompatibility or CI) (Barr, 1980; Breeuwer et al., 1992; O’Neill et al., 1992; Rousset et al., 1992), parthenogenesis (Stouthamer et al., 1993) and feminization of genetic males (Rousset $e t$ al., 1992).

Cytoplasmic incompatibility, which was originally discovered in mosquitoes (Laven, 1957; Barr, 1980), is manifested as partial to complete embryonic death in crosses between certain strains. The factors responsible for CI were shown to be cytoplasmically (mater-

*Correspondence. nally) inherited. Subsequent research in a number of different species has shown that $\mathrm{CI}$ is caused by intracellular bacteria found in the reproductive tissues.

Bacterially induced cytoplasmic incompatibility is of interest as a potentially rapid mechanism for development of reproductive isolation and subsequent speciation (Breeuwer \& Werren, 1990; Coyne, 1992). Consistent with this possibility, bidirectional incompatibility has been found between geographical races of Culex pipiens, between different geographical isolates of Drosophila simulans (O'Neill \& Karr, 1990) and between closely related species of Nasonia wasps (Breeuwer \& Werren 1990; Breeuwer, 1993). To determine the possible role of these bacteria in facilitating speciation events, the extent of association of the bacteria with reproductive incompatibility between closely related species must be determined.

Demonstration of a definitive association between Wolbachia and CI includes showing that: (i) Wolbachia are present in at least one strain or species, (ii) antibiotic curing eliminates the Wolbachia, and (iii) subse- 
quent crosses between cured and uncured strains demonstrate unidirectional incompatibility: Although the precise mechanisms of $\mathrm{CI}$ are unknown, cytogenetic studies in the parasitic wasp Nasonia vitripennis indicate that $\mathrm{CI}$ is caused by improper condensation and fragmentation of the paternal chromosomes in the first mitotic division of the fertilized egg (Ryan \& Saul, 1968; Breeuwer \& Werren, 1990; Reed \& Werren, 1995). Similar abnormal mitoses have been observed in Drosophila simulans infections, indicating that the same mechanism may be operating in diverse species. In haplodiploid species, such as $N$. vitripennis, paternal genome loss typically results in production of male (haploid) offspring, whereas in diplodiploids it results in zygotic lethality.

Although Wolbachia are widespread in insects (Werren et al., 1995; R. Giodano \& H. M. Robertson, personal communication), occurring in 5-10 per cent of species examined, Wolbachia-associated cytoplasmic incompatibility has only been definitively demonstrated in a small number of species, including several Diptera (Culex pipiens, Drosophila simulans and D. melanogaster), two Coleoptera (Tribolium confusum and Hypera postica), one Homoptera (Laedophax striatellus), one Lepidoptera (Ephestia cautella) and three Hymenoptera (Nasonia vitripennis, $N$. giraulti and $N$. longicornis). Many of the species in which $\mathrm{CI}$ has been demonstrated have recently achieved cosmopolitan distributions and thus may not represent populations at evolutionary equilibrium. For example, T. confusum and E. cautella are associated with stored grain products. The cosmopolitan species $C$. pipiens, D. melanogaster, $D$. simulans and $N$. vitripennis have widespread distributions, apparently caused at least in part by recent range expansion as a result of human activities. $H$. postica and $L$. striatellus are insect pests associated with human agriculture.

Here we focus on the presence of Wolbachia and its role in $\mathrm{CI}$ in several endemic species belonging to the closely related quinaria, testacea and tripunctata groups of Drosophila. There is no evidence that the geographical ranges and breeding biology of these woodland species have been strongly altered by human activity. Each of these species is endemic to a particular geographical region in either North America or Eurasia.

\section{Methods}

\section{Insect strains}

Assays for the presence of Wolbachia were carried out in 10 species of Drosophila belonging to the closely related quinaria, testacea and tripunctata species groups of the subgenus Drosophila. The species and the origin, including date of collection, of the strains used are as follows: quinaria group members $D$. fallen $i$ (Rochester, New York, 1992), D. recens (two strains from the White Mountains, New Hampshire, 1993; three strains from Rochester, 1992 (1) and 1993 (2)), D. quinaria (Rochester, 1993), D. palustris (Rochester, 1993 ) and $D$. phalerata (Leiden, Netherlands; Regensburg, Germany, 1990); testacea group members $D$. testacea (Regensburg, 1990), D. neotestacea (Rochester, 1992), D. orientacea (Sapporo, Japan, 1990) and D. putrida (Rochester, 1992); and D. tripunctata (Great Smoky Mountains, Tennessee, 1987) of the tripunctata group. These strains had been maintained in the laboratory at $21^{\circ} \mathrm{C}$ on Instant Drosophila Medium (Carolina Biological Supply) plus commercial Agaricus bisporus mushrooms. All of the species breed primarily on mushrooms in the wild, except $D$. quinaria and $D$. palustris, which breed on decaying skunk cabbages (Symplocarpus foetidus). $D$. falleni, D. recens, $D$. quinaria, D. palustris, $D$. putrida, $D$. neotestacea and $D$. tripunctata are endemic to North America. D. phalerata and D. testacea are known from Europe and continental Asia, and $D$. orientacea is endemic to Japan.

\section{PCR assay}

DNA from each strain was prepared from four to 10 ovaries of fecund females. Ovaries were dissected in sterile double-distilled deionized water on a sterile slide and then serially transferred to droplets of sterile $\mathrm{H}_{2} \mathrm{O}$ prior to transfer into a $0.5 \mathrm{~mL}$ microfuge tube containing $0.3 \mathrm{~mL}$ sterile zirconium beads (Biospec Products, Bartlesville, OK), $200 \mu \mathrm{L}$ Tris, $10 \mu \mathrm{L} 20$ per cent SDS and $200 \mu \mathrm{L}$ phenol. Samples were shaken in a mini-Bead beater (Biospec Products) for $3 \mathrm{~min}$. Phases were separated by $10 \mathrm{~min}$ spinning in a microcentrifuge. The DNA was then ethanol precipitated and resuspended in $20 \mu \mathrm{L}$ sterile TE buffer $(10 \mathrm{~mm}$ Tris ( $\mathrm{pH} \mathrm{8),} 1$ mм EDTA). Extreme care was taken throughout the procedure to avoid bacterial contamination. All solutions were filter sterilized $(0.22 \mu \mathrm{m}$ pore size). Control DNA samples were prepared using pupae of known infected and uninfected strains of the parasitoid wasp, Nasonia vitripennis.

Bacterial $f t s Z$ DNA was amplified using PCR in a volume of $25 \mu \mathrm{L}(1 \mu \mathrm{L}$ DNA sample, $2.5 \mu \mathrm{L}$ $10 \times$ buffer (Promega), $0.75 \mu \mathrm{L} \mathrm{MgCl}_{2}(50 \mathrm{~mm}), 0.5 \mu \mathrm{L}$ nucleotide mix ( $10 \mathrm{mM}$ each), $0.350 \mu \mathrm{L} 20 \mathrm{~mm}$ primer $1,0.350 \mu \mathrm{L} 20 \mathrm{~mm}$ primer $2,0.25 \mu \mathrm{L}$, Taq polymerase (Promega) and double-distilled $\mathrm{H}_{2} \mathrm{O}$ was added to a final volume of $25 \mu \mathrm{L}$ ). Two primer pairs designed for amplification of Wolbachia ftsZ sequences were used, 
ftsZf1 $\quad 5^{\prime}$-GTTGTCGCAAATACCGATGC- $3^{\prime}$ and ftsZr1 5'-CTTAAGTAAGCTGGTATATC-3'. These yield an amplification product of 1035-1047 bp.

The PCR reaction mix was prepared in one batch and then added to each sample. The remainder was run as a control for contamination. PCR cycling conditions were one cycle $\left(1 \mathrm{~min}\right.$ at $94^{\circ} \mathrm{C}, 1 \mathrm{~min}$ at $55^{\circ} \mathrm{C}, 3 \mathrm{~min}$ at $\left.72^{\circ} \mathrm{C}\right), 35$ cycles $\left(15 \mathrm{~s}\right.$ at $94^{\circ} \mathrm{C}, 1 \mathrm{~min}$ at $55^{\circ} \mathrm{C}, 3 \mathrm{~min}$ at $\left.72^{\circ} \mathrm{C}\right)$ and 1 cycle $\left(15 \mathrm{~s}\right.$ at $94^{\circ} \mathrm{C}, 1 \mathrm{~min}$ at $55^{\circ} \mathrm{C}$ and 10 min at $72^{\circ} \mathrm{C}$ ) (Erincomp thermal cycler). After PCR, 1 $\mu \mathrm{L}$ of amplified reaction product was run on a 1 per cent agarose gel to determine presence and size of amplified DNA.

\section{Cloning and sequencing}

For cloning purposes, a $50 \mu \mathrm{L}$ PCR reaction was used (doubling of solutions above) for amplification of the two known positives, $D$. recens and $D$. orientacea. PCR products were purified with the Geneclean kit (Bio 101, La Jolla, CA) and dissolved in $20 \mu \mathrm{L}$ distilled deionized $\mathrm{H}_{2} \mathrm{O}$, and then directly cloned into a T-tailed M13mp18 derivative (W. Burke, unpublished data). Two clones each were sequenced for $D$. recens and $D$. orientacea. Two regions of ambiguity in the sequences caused by apparent secondary structure effects were resolved by additional sequencing reactions using the modified base analogue dITP.

\section{Antibiotic curing}

A strain of $D$. recens collected in 1993 in the White Mountains of New Hampshire was cured of its symbiotic Wolbachia as follows. Fresh Agaricus bisporus mushrooms were soaked to saturation in a solution of tetracycline $(0.1,0.25,1.0$ or $2.0 \mathrm{mg}$ tetracycline per $\mathrm{mL}$ ). These mushrooms were then placed in vials with 10 males and 10 females of $D$. recens at $22^{\circ} \mathrm{C}$. Flies were allowed to oviposit on the mushrooms for 6 days. Tetracycline solution of the appropriate strength was added on the sixth day to the mushrooms to keep them saturated; at this point all of the cultures had first and second instar larvae actively feeding. Upon emergence of adults, flies were kept on instant plus mushroom medium without tetracycline. Treated strains were tested using the PCR assay to determine if they harbored detectable levels of Wolbachia.

\section{Crossing experiments}

The effect of the Wolbachia on cytoplasmic incompatibility was tested twice, once after two generations of maintenance on tetracycline-free medium, and once after three generations on this medium. Compatibility was assayed by carrying out crosses between single males and single females. For each tetracycline concentration in which the larvae were initially grown $(0.1$, $0.25,1.0$, and $2.0 \mathrm{mg} / \mathrm{mL}$ ), reciprocal crosses were carried out with flies of an untreated, Wolbachia-infected strain. In addition, crosses were carried out between treated males and treated females for each tetracycline treatment. Ten replicates of each cross were used. Adults were left in their culture vials for 14 days in the first trial and 7 days in the second.

Preliminary molecular phylogenetic analysis indicates that $D$. quinaria is more closely related to $D$. recens than any other quinaria group species we examined (G. Spicer, personal communication). To determine whether Wolbachia play a role in the reproductive isolation between these sympatric species, we carried out reciprocal crosses between $D$. quinaria and either Wolbachia-positive or Wolbachia-negative strains of $D$. recens. For each cross type, five males and five females were placed in each of three vials. These were watched for $5 \mathrm{~h}$ to determine if any copulation took place. The flies were left in the vials and the cultures were kept at $22^{\circ} \mathrm{C}$. The cultures were thoroughly examined under a microscope $(12 \times)$ to see if any viable larvae were produced in any of the cultures.

\section{Statistical analysis}

An effect of Wolbachia on cytoplasmic incompatibility would be manifest by inequalities in offspring production between the reciprocal crosses between Wolbachia-positive and Wolbachia-negative flies. For each tetracycline concentration, the numbers of offspring produced per pair were analysed for three cross types, where I (Infected) refers to the untreated Wolbachia-infected strain and $\mathrm{T}$ (Treated) refers to flies treated with tetracycline: $\mathrm{T}$ female $\times \mathrm{I}$ male, $\mathrm{I}$ female $\times \mathrm{T}$ male, and $\mathrm{T}$ female $\times \mathrm{T}$ male. Crosses between treated males and females were included in the analysis to test the possibility that reduced offspring production in the $T$ female $\times I$ male crosses was because of reduced fertility of $\mathrm{T}$ females, rather than of cytoplasmic incompatibility. An analysis of variance (PROC ANOVA, SAS Institute, 1990) was used to determine if there was heterogeneity among cross types in offspring production. If there was significant variation, then a Fisher's Least Significant Difference test (LSD) was carried out to determine which cross type(s) produced fewer offspring than the others.

\section{Results}

To screen for the presence of Wolbachia by PCR, primers were utilized that amplify the bacterial fts $\mathrm{Z}$ 
gene (Werren et al., 1995). Sequencing of fts $\mathrm{Z}$ product from over 25 different insect species has revealed that these primers are highly specific for Wolbachia (no other bacterial genera have been sequenced from amplification products using these primers) and that the primers amplify both of the two major subdivisions of Wolbachia (Werren et al., 1995). Table 1 shows the results of attempts to amplify Wolbachia ftsZ product from ovaries of 10 species of Drosophila. Each strain was examined at least twice, with the flies used for DNA isolation in the different replicates having been reared separately. Two species, $D$. recens and $D$. orientacea, were found to be positive for Wolbachia. PCR reactions from both yielded an amplification product of the expected size, 1035-1047 bases.

Following the positive result from $D$. recens, four additional strains of this species were tested. Two of these had recently been collected from field populations in upstate New York and two from New Hampshire. All five strains of $D$. recens tested positive for Wolbachia, indicating that these bacteria are widespread and common in this species. Additional strains of $D$. orientacea were not available for analysis.

Amplification products from $D$. recens and $D$. orientacea were cloned into an M13 derivative and sequenced. A phylogenetic analysis utilizing these

Table 1 Results of PCR amplification of Wolbachia ftsZ sequences in 10 species of Drosophila

\begin{tabular}{|c|c|c|c|}
\hline Species & Strain & $\begin{array}{l}\text { No. of } \\
\text { replicates }\end{array}$ & $\begin{array}{c}f t s \mathrm{Z} \\
\text { sequences } \dagger\end{array}$ \\
\hline falleni & Rochester & 2 & Absent \\
\hline quinaria & Rochester & 2 & Absent \\
\hline \multirow[t]{6}{*}{ recens } & Mendon 1992 & 3 & Present \\
\hline & NH-1 & $2 \ddagger$ & Present \\
\hline & NH-2 & $2 \ddagger$ & Present \\
\hline & Rochester-1 & $2 \ddagger$ & Present \\
\hline & Rochester-2 & $2 \ddagger$ & Present \\
\hline & Treated & 2 & Absent \\
\hline palustris & Rochester & 2 & Absent \\
\hline \multirow[t]{2}{*}{ phalerata } & Regensburg, Germany & 2 & Absent \\
\hline & Leiden, Netherlands & 2 & Absent \\
\hline tripunctata & $\begin{array}{l}\text { Great Smoky Mtns, } \\
\text { TN }\end{array}$ & 2 & Absent \\
\hline putrida & Rochester & 2 & Absent \\
\hline neotestacea & Rochester & 3 & Absent \\
\hline testacea & Regensburg, Germany & 3 & Absent \\
\hline orientacea & Sapporo, Japan & 3 & Present \\
\hline
\end{tabular}

$\uparrow$ All replicates for each strain yielded identical results. Replicates represent independent isolations of DNA and PCR amplification, except for the cases marked $\ddagger$ which were separate amplifications from the same DNA isolation. sequences and a representative sample from 25 other species was performed (data not shown). The analysis was performed using the neighbour-joining algorithm following determination of Jukes \& Cantor (1969) genetic distances. Wolbachia from both $D$. recens and $D$. orientacea belong to the $\mathrm{Dm}$ clade of the A subdivision of these bacteria and are thus referred to as Adm. The Adm 'strain' of Wolbachia is a subgroup that shows a high degree of sequence similarity but is found in a diverse range of insect hosts (including Diptera, Hymenoptera and Lepidoptera) (Werren et al., 1995). This pattern indicates that this strain of Wolbachia has been undergoing considerable horizontal transmission between insects, accounting for the low amount of sequence divergence. This strain of Wolbachia is also found in other drosophilid species, including $D$. melanogaster and D. simulans.

The results of crosses between treated (descendants of tetracycline-treated larvae) and untreated flies are presented in Table 2. It should be noted that the treated strain was derived by antibiotic treatment two to three generations previously from the same infected strain used in these crosses. As observed in other known cases of cytoplasmic incompatibility in Diptera, crosses between infected males and cured females in D. recens yielded significantly fewer adult progeny than the reciprocal crosses. This effect was observed in both replicate trials, which were carried out in sequential generations. By the criterion of incompatibility, a single generation of larval development in mushrooms treated with $1.0,2.0$ and probably $0.25 \mathrm{mg}$ of tetracycline per $\mathrm{mL}$ was sufficient to produce at least partial cytoplasmic incompatibility. PCR analysis also indicated that these strains were free of Wolbachia. Development in mushrooms soaked the lowest concentration $(0.1 \mathrm{mg} / \mathrm{mL})$ of tetracycline apparently was not effective in curing the flies of Wolbachia.

Table 2 also shows that the crosses between treated males and treated females produced offspring numbers comparable to the compatible crosses between infected females and treated males. This shows that the significantly lower offspring production in the crosses between infected males and treated females is not the result of reduced fertility of treated females.

In the interspecific crosses between $D$. recens (both cured and infected) and $D$. quinaria, which does not harbour Wolbachia (see Table 1), at least one interspecific mating was seen during the 5-h observation period for each of the following cross types: $D$. quinaria male $\times D$. recens female (cured), $D$. recens male (cured) $\times D$, quinaria female, and $D$. recens male (infected) $\times D$. quinaria female. No larvae were observed in any of the cultures for any of the interspecific crosses. This indicates that there is complete 
Table 2 Mean $( \pm \mathrm{SE})$ number of offspring produced in Drosophila recens crosses

\begin{tabular}{|c|c|c|c|c|c|c|}
\hline \multirow{2}{*}{\multicolumn{2}{|c|}{ Tetracycline treatment $(\mathrm{mg} / \mathrm{mL})$}} & \multicolumn{4}{|c|}{ Cross type $(\text { female } \times \text { male })^{*}$} & \multirow[b]{2}{*}{ Probability } \\
\hline & & \multirow{2}{*}{$\begin{array}{c}\mathrm{I} \times \mathrm{I} \\
58.1 \pm 10.0\end{array}$} & \multirow{2}{*}{$\frac{\mathrm{T} \times \mathrm{I}}{38.1 \pm 14.6^{\mathrm{a}}}$} & \multirow{2}{*}{$\frac{\mathrm{I} \times \mathrm{T}}{36.3 \pm 13.6^{\mathrm{a}}}$} & \multirow{2}{*}{$\frac{\mathrm{T} \times \mathrm{T}}{63.6 \pm 13.9^{\mathrm{a}}}$} & \\
\hline Trial 1 & 0.1 & & & & & 0.28 \\
\hline & 0.25 & $58.1 \pm 10.0$ & $26.8 \pm 17.1^{\mathrm{a}, \mathrm{b}}$ & $22.8 \pm 9.0^{\mathrm{a}}$ & $71.6 \pm 19.7^{b}$ & 0.07 \\
\hline & 1.0 & $58.1 \pm 10.0$ & $13.0 \pm 10.0^{\mathrm{a}}$ & $58.7 \pm 16.2^{b}$ & $51.7 \pm 7.3^{b}$ & 0.024 \\
\hline & 2.0 & $58.1 \pm 10.0$ & $3.6 \pm 2.1^{\mathrm{a}}$ & $64.6 \pm 20.5^{b}$ & $42.8 \pm 14.7^{\mathrm{a}, \mathrm{b}}$ & 0.019 \\
\hline Trial 2 & 0.1 & $38.8 \pm 12.2$ & $34.3 \pm 9.2^{\mathrm{a}}$ & $30.1 \pm 11.4^{\mathrm{a}}$ & $53.6 \pm 14.6^{\mathrm{a}}$ & 0.35 \\
\hline & 0.25 & $38.8 \pm 12.2$ & $3.5 \pm 1.6^{\mathrm{a}}$ & $49.9 \pm 13.8^{b}$ & $31.9 \pm 8.8^{b}$ & 0.007 \\
\hline & 1.0 & $38.8 \pm 12.2$ & $1.7 \pm 1.5^{\mathrm{a}}$ & $35.9 \pm 6.3^{b}$ & $29.0 \pm 9.7^{b}$ & 0.003 \\
\hline & 2.0 & $38.8 \pm 12.2$ & $1.4 \pm 0.9^{\mathrm{a}}$ & $22.7 \pm 6.8^{\mathrm{b}}$ & $35.4 \pm 10.4^{b}$ & 0.008 \\
\hline
\end{tabular}

T: flies descended from tetracycline-treated larvae; I: flies infected with Wolbachia (untreated).

Trial 1 was performed two generations after antibiotic treatment; trial 2 was carried out the following generation.

Probability values are those obtained by analysis of variance of offspring numbers within rows.

*Entries within rows marked with the same superscript ( $\mathrm{a}$ or $\mathrm{b}$ ) are not significantly different $(P<0.05)$ by a least significant difference (LSD) test (SAS Institute, 1990). I $\times$ I crosses were not included in ANOVA S or LSD tests because only one set of 10 crosses per trial was carried out.

post-mating reproductive isolation between these species and that curing $D$. recens of Wolbachia does not confer reproductive compatibility between this species and $D$. quinaria. The result indicates that Wolbachia are not involved in reproductive isolation between these species.

\section{Discussion}

Of the 10 species of Drosophila tested, two (D. recens and $D$. orientacea) were found to be positive for Wolbachia. All five strains of $D$. recens tested were found to be infected with Wolbachia. Thus, Wolbachia infection appears to be common in this species. Because only one strain of $D$. orientacea was available for screening, we cannot infer the prevalence of infection.

It should be emphasized that a negative PCR result does not establish that a species is uninfected. Some strains have been kept under laboratory rearing conditions for several years, and could have lost Wolbachia during this time. In addition, individual species may be polymorphic for Wolbachia infections in natural populations. For example, studies of $D$. melanogaster in Australia reveal polymorphisms for Wolbachia infections in several different populations (Hoffmann, 1988; Solignac et al., 1994). Similarly, D. simulans is polymorphic for Wolbachia, although studies indicate that the infection is rapidly spreading through that species (Hoffmann et al., 1986; Turelli \& Hoffmann, 1991). Some theoretical studies predict that polymorphisms for cytoplasmic incompatibility Wolbachia infections will often be transient, with most infections eventually spreading to fixation in the population (Caspari \& Watson, 1959). However, this prediction is based on the assumption of complete maternal transmission of the infection to progeny. Polymorphism for infection is predicted under a variety of conditions (Rousset $e t$ al., 1991; Stevens \& Wicklow, 1992; Wade \& Stevens, 1994; Turelli, 1995).

Several of the insects in which Wolbachia cause cytoplasmic incompatibility are cosmopolitan in distribution, having undergone dramatic range increases within historical times. These include $D$. melanogaster, D. simulans, Culex pipiens, Tribolium confusum, Nasonia vitripennis and Ephestia cautella. It is therefore possible that acquisition of Wolbachia infections is associated with range expansion or occupation of disturbed habitats. In contrast, $D$. recens is endemic to forests of northeastern North America (Wheeler, 1960). It breeds only in natural substrates, primarily mushrooms, and is not associated with humans. Similarly, $D$. orientacea is endemic to natural habitats in Japan. Our results with $D$. recens and $D$. orientacea argue that rapid range expansion and association with disturbed environments is not a requirement for acquisition of Wolbachia. It will be worthwhile to determine whether the incidence of Wolbachia infection is greater in cosmopolitan species than in those that have not undergone recent range expansions.

Although Wolbachia are typically transmitted vertically within the egg cytoplasm of their host species, it is clear from molecular phylogenetic studies that hori- 
zontal transmission of these bacteria can occur, even between different orders of insects (O'Neill et al., 1992; Stouthamer et al., 1993; Werren et al., 1995). The mechanisms of this horizontal transmission are unknown, although transmission via associated parasitoid insects has been postulated as one possibility. Werren et al. (1995) present phylogenetic evidence that such transfer has occurred between parasitic wasps of the genus Nasonia and their blowfly hosts, Protocalliphora. Recent experiments using microinjection techniques to move Wolbachia between insect species also show that Wolbachia strains can become established in different species and still cause incompatibility (Boyle et al., 1993; Braig et al., 1994; Chang \& Wade, 1994). Presumably, occasional natural transmission of these bacteria across species boundaries can result in subsequent expansion of the infection and associated cytoplasmic incompatibility in the new host species.

The 10 drosophilid species screened for Wolbachia are interesting because, except for $D$. quinaria and $D$. palustris, they share a common ecology, the mushroom-feeding habit. Several of these mycophagous species, including $D$. falleni, $D$. putrida, $D$. neotestacea and $D$. tripunctata, are sympatric with $D$. recens over much of their range in eastern North America. Furthermore, these species are normally found associated on individual mushrooms (Jaenike \& James, 1991): mushrooms yielding many individuals of $D$. recens, which is infected with Wolbachia, typically yield numerous individuals of the other species. Finally, the phylogenetic positions of the Wolbachia found in $D$. recens (and $D$. orientacea) indicate that these flies obtained their resident Wolbachia via horizontal transfer from other host taxa. Thus, there would appear to be ample ecological opportunity for horizontal spread of Wolbachia from $D$. recens to the other mycophagous drosophilids. It is interesting that $D$. recens harbours these bacteria while closely related and ecologically associated drosophilids apparently do not. Similarly, Hoffmann et al. (1994) found that many Australian populations of $D$. melanogaster are infected with $\mathrm{CI}$ Wolbachia, whereas microsympatric $D$. simulans populations appear to be uninfected. This pattern suggests that horizontal transfer is a rare event or is typically not mediated directly through a shared feeding substrate.

We postulate that the frequency of infected species results from a balance between rates of (i) horizontal transmission to uninfected species, (ii) loss of Wolbachia from infected species, and (iii) speciation and extinction of infected and uninfected species. That relatively few insects harbour Wolbachia (5-10 per cent) could be explained in a variety of ways. For instance, infected species may experience greater rates of extinc- tion, although this could be offset by greater rates of speciation, as Wolbachia may increase speciation rates by providing an additional mechanism for reproductive isolation, namely cytoplasmic incompatibility. Mechanisms of loss in natural populations include the evolution of resistance (Turelli, 1995), reduced fitness of infected individuals, natural curing (Stevens \& Wickow, 1992), and stochastic loss of bacteria within infected individuals.

It may be a coincidence, but the two species we found to be infected with Wolbachia, D. recens and $D$. orientacea, have the most northerly distributions of the 10 species we examined (Wheeler, 1960; Grimaldi et al., 1992). It would be interesting to determine if an endemic species with a broad latitudinal range exhibited geographical variation in the prevalence of infection. It would also be worthwhile to determine in the laboratory if reductions in host fitness or rates of spontaneous loss of symbionts were temperaturedependent.

Evidence suggests that at least one strain of these bacteria (the Adm strain) is undergoing considerable recent horizontal transmission (Werren et al., 1995). This conclusion is based on molecular phylogenetic data (Werren et al., 1995) and the observation that this strain is currently sweeping through $D$. simulans (Turelli \& Hoffmann, 1991); it may have been recently acquired by $D$. melanogaster as well (Hoffmann, 1988; Solignac et al., 1994). D. recens and D. orientacea also harbour the Adm strain of Wolbachia. Because these two species, unlike $D$. melanogaster and $D$. simulans, are not associated with human-disturbed habitats, the finding that it is infected with the Adm strain of Wolbachia suggests that these bacteria are an integral part of natural as well as disturbed ecological communities. Analysis of the ecological and phylogenetic patterns of association of hosts and Wolbachia could provide useful insights into the primary mode of horizontal transmission of these bacteria.

\section{Acknowledgments}

We thank Wan Zhang, Julie Chen and Li Rong Guo for PCR, cloning and sequencing aspects of this study. This research was supported by grants from the National Science Foundation to J.W. (DEB-9220652 and DEB931 8783) and J.J. (DEB-9206916).

\section{References}

BARR, A. R. 1980. Cytoplasmic incompatibility in natural populations of a mosquito, Culex pipiens L. Nature, 283, $71-72$. 
BOYLE, L., O'NEILL, S. L., ROBERTSON, H. AND KARR, T. L. 1993. Horizontal transfer of Wolbachia pipientis by microinjection of egg cytoplasm: infection levels and the expression of cytoplasmic incompatibility in Drosophila. Science, 260, 1796-1799.

BRAIG, H. R., GUZMAN H., TESH, R. B. AND O'NEILL, S. L. 1994. Replacement of the natural Wolbachia symbiont of Drosophila simulans with a mosquito counterpart. Nature, 367 , 453-455.

BREEUWer, J. A. J. 1993. Postzygotic Reproductive Isolation in the Genus Nasonia (Hymenoptera, Pteromalidae): the Role of Cytoplasmic Microorganisms and Other Heritable Cytoplasmic Factors. Ph. D. Thesis, University of Rochester, Rochester, New York.

BREEUWER J. A. J., STOUTHAMER, R., BARNS, S. M., PELl.ETIER, D. A., WEISBURG, W. G. AND WERREN, J. H. 1992. Phylogeny of cytoplasmic incompatibility micro-organisms in the parasitoid wasp genus Nasonia (Hymenoptera: Pteromalidae) based on $16 \mathrm{~S}$ ribosomal DNA sequences. Insect Mol. Biol., 1, 25-36.

BREEUWER, J. A. J. AND WERREN, J. H. 1990. Microorganisms associated with chromosome destruction and reproductive isolation between two insect species. Nature, 346, $558-560$.

CASPARI, E. AND WATSON, G. S. 1959. On the evolutionary importance of cytoplasmic sterility in mosquitoes. Evolution, 13, 568-570.

CHANG, N. W. AND WADE, M. J. 1994. The transfer of Wolbachia pipientis and reproductive incompatibility between infected and uninfected strains of the flour beetle, Tribolium confusum, by microinjection. Can. J. Microbiol., 40, 978-981.

CoYNE, J. A. 1992. Genetics and speciation. Nature, 355, 511-515.

GRIMALDI, D. A., JAMES, A. C. AND JAENIKE, J. 1992. Systematics and modes of reproductive isolation in the Drosophila testacea group (Diptera: Drosophilidae). Ann. Entomol. Soc. Am., 85, 671-685.

HoffmanN, A. A. 1988. Partial cytoplasmic incompatibility between two Australian populations of Drosophila melanogaster. Entomologia exp. appl., 48, 61-67.

HOFFMANN A. A., TURELLl, M. AND SIMMONS, G. M. 1986. Unidirectional incompatibility between populations of Drosophila simulans. Evolution, 40, 692-701.

JAEnjKe, J. AND JAMES, A. C. 1991. Aggregation and the coexistence of mycophagous Drosophila. J. Anim. Ecol., 60, 913-928.

JUKES, T. H. AND CANTOR, C. R. 1969. Evolution of protein molecules. In: Munro, H. (ed.) Mammalian Protein Evolution, pp. 21-132. Academic Press, New York.

LAVEN, H. 1957. Verebung durch Kerngene und das Problem der ausserkaryotischen Vererbung bei Culex pipiens. II. Ausserkaryotische Vererbung. Z. Indukt. Abst. U. Vererb., 88, 478-516.

O'NEILL, S. L., GIORDANO, R., COLBERT, A. M. E., KARR, T. L. AND ROBERTSON, H. M. 1992. 16S rRNA phylogenetic analysis of the bacterial endosymbionts associated with cytoplasmic incompatibility in insects. Proc. Natl. Acad. Sci. U.S.A., 89, 2699-2702.

O'NEILL, S. L. AND KARR, T. L. 1990. Bidirectional incompatibility between conspecific populations of Drosophila simulans. Nature, 348, 178-180.

REED, K. AND WERREN, J. W. 1995. Induction of paternal genome loss by the paternal sex ratio chromosome and cytoplasmic incompatibility bacteria (Wolbachia): a comparison of early embryonic events. Mol. Reprod. Dev., 40, 408-418.

RousSET, F., BOUCHON, D., PINTUREAU, B., JUChaUlt, P. AND SOLJGNAC, M. 1992. Wolbachia endosymbionts responsible for various alterations of sexuality in arthropods. Proc. $R$. Soc. $B, 250,91-98$.

ROUSSET, F., RAYMOND, M. AND KJELLLBERG, F. 1991. Cytoplasmic incompatibilities in the mosquito Culex pipiens: how to explain a cytotype polymorphism? J. Evol. Biol., 4, 69-81.

RYAN, S. L. AND SAUL, G. B., 2nd 1968. Post-fertilization effect of incompatibility factors in Mormoniella. Mol. Gen. Genet., 103, 29-36.

SAS INSTITUTE. 1990. SAS/STAT User's guide, Version 6, vol. 2. SAS Institute, Cary, NC.

SOLIGNAC, M., VAUTRIN, D. AND ROUSSET, F. 1994. Widespread occurrence of the proteobacteria Wolbachia and partial cytoplasmic incompatibility in Drosophila melanogaster. C. r. Acad. Sci. Paris (Life Sciences), 317, 461-470.

STEVENS, L. AND WICKLow, D. T. 1992. Multispecies interactions affect cytoplasmic incompatibility in Tribolium flour beetles. Am. Nat., 140, 642-653.

STOUTHAMER, R., BREEUWER, J. A. J., LUCK, R. F. AND WERREN, J. H. 1993. Molecular identification of microorganisms associated with parthenogenesis. Nature, 361, 66-68.

TURELLL1, M. 1995. Evolution of incompatibility-inducing microbes and their hosts. Evolution (in press).

TURELlı, M. AND HOFFMANN, A. A. 1991. Rapid spread of an incompatibility factor among natural Drosophila populations. Nature, 353, 440-442.

WADE, M. J. AND STEVENS, L. 1994. The effect of population subdivision on the rate of spread of parasite-mediated cytoplasmic incompatibility. J. Theor. Biol., 167, 81-87.

WERREN, J. H., ZHANG, W. AND GUO, L. R. 1995. Phylogeny of Wolbachia bacteria: reproductive parasites of arthropods. Proc. $R$. Soc. $B$. (in press).

WHEELER, M. R. 1960. New species of the quinaria group of Drosophila (Diptera, Drosophilidae). Southwestern Naturalist, 5, 160-164. 